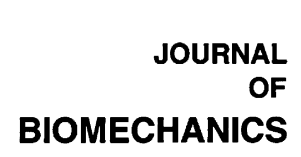

Journal of Biomechanics 40 (2007) 2655-2662

www.elsevier.com/locate/jbiomech www.JBiomech.com

\title{
Failure properties and strain distribution analysis of meniscal attachments
}

\author{
Diego F. Villegas, Jason A. Maes, Sarah D. Magee, Tammy L. Haut Donahue* \\ Department of Mechanical Engineering-Engineering Mechanics, Michigan Technological University, MI, USA
}

Accepted 8 January 2007

\begin{abstract}
The menisci are frequently injured due to both degeneration and traumatic tearing. It has been suggested that the success of a meniscal replacement is dependent on several factors, one of which is the secure fixation and firm attachment of the replacement to the tibial plateau. Therefore, the objectives of the current study were to (1) determine the failure properties of the meniscal horn attachments, and (2) determine the strain distribution over their surfaces. Eight bovine knee joints were used to study the mechanical response of the meniscal attachments. Three meniscal attachments from one knee of each animal were tested in uniaxial tension at $2 \% / \mathrm{s}$ to determine the load deformation response. During the tests, the samples were marked and local strain distributions were determined with a video extensometer. The linear modulus of the medial anterior attachment $(154 \pm 134 \mathrm{MPa})$ was significantly less than both the medial posterior $(248 \pm 179 \mathrm{MPa}, p=0.0111)$ and the lateral anterior attachment $(281 \pm 214 \mathrm{MPa}, p=0.0007)$. Likewise, the ultimate strain for the medial anterior attachments $(13.5 \pm 8.8 \%)$ was significantly less than the medial posterior $(23 \pm 13 \%, p<0.0001)$ and the lateral anterior attachment $(20.3 \pm 11.1 \%, p=0.0033)$. There were no significant differences in the structural properties or ultimate stress between the meniscal attachments $(p>0.05)$. No significant differences in ultimate strain or moduli across the surface of the attachments were noted. Based on the data obtained, a meniscal replacement would need different moduli for each of the different attachments. However, the attachments appear to be homogeneous.
\end{abstract}

(C) 2007 Elsevier Ltd. All rights reserved.

KeyWords: Meniscus; Knee; Material properties; Meniscal replacement

\section{Introduction}

The menisci perform a variety of functions within the knee, but their most prevalent role is in weight bearing and load transmission across the knee joint (Morrison, 1970; Walker and Erkman, 1975; Shrive et al., 1978; Ghosh and Taylor, 1987; Renstrom and Johnson, 1990; Ahmed, 1992; Messner and Gao, 1998). The menisci are able to carry out this function due to their structural shape and firm attachment to the tibia (Shrive et al., 1978; Fithian et al., 1990; Renstrom and Johnson, 1990; Gao et al., 1998; Messner and Gao, 1998). When a meniscus is injured, two options are available to repair the damaged meniscus: surgical repair of the meniscal tear or a partial or full menisectomy (Ghadially et al., 1986; DeHaven, 1992;

*Corresponding author. Tel.: + 19064872078 ; fax: + 19064872822.

E-mail address: thdonahu@mtu.edu (T.L. Haut Donahue).
Newman et al., 1993; Asik and Sener, 2002). In the latter case, the procedure has been shown to lead to degeneration of the articular cartilage of the knee (Allen et al., 1984; McBride and Reid, 1988; Moon et al., 1988; Messner, 1999; Rodeo, 2001; Wyland et al., 2002). Therefore, if the meniscus must be removed, a sound option for its replacement must be readily available that can duplicate its biomechanical function.

While the material properties of meniscal tissue have previously been studied, meniscal attachments have received little attention. It has been shown that the meniscal attachments are important for restoring functionality to the knee (Chen et al., 1996; Goertzen et al., 1996; Paletta et al., 1997; Alhalki et al., 1999; Rodeo, 2001; Sekaran et al., 2002; Haut Donahue et al., 2003). Therefore, their time-dependent and failure properties need to be determined. We have already obtained the time-dependent properties of the meniscal attachments and found no 
significant differences in creep or stress relaxation properties between the anterior and posterior attachments of the medial meniscus. However, there were significant differences in creep parameters between the lateral anterior attachment and the medial attachments (Maes and Haut Donahue, 2006). One other study has documented the peak failure loads of rabbit meniscal attachments with no values provided for the material properties (Goertzen et al., 1996). Therefore, study of the attachment mechanisms must be conducted to further the development of a successful meniscal replacement. The objectives of the present study are to (1) determine the failure properties of the meniscal horn attachments, and (2) determine the strain distribution over their surfaces.

\section{Methods}

\subsection{Specimen preparation}

Eight bovine stifle (knee) joints with an age range between 15 and 30 months were obtained from a slaughterhouse and frozen at $-20^{\circ} \mathrm{C}$ until the day of testing. From each animal, either the right knee or the left knee was randomly chosen for inclusion in the study. No attempt was made to study the differences between contralateral knees. On the day of testing, specimens were thawed at room temperature and disarticulated. All tissues were removed leaving only the proximal tibia with the menisci and their attachments intact. The attachments were cut leaving approximately $7 \mathrm{~mm}$ of the central third of the attachment intact. The tibia was cut around the insertion sites of the attachments leaving small bone blocks fastened to the meniscal horns. The bone blocks were potted within a custom fixture using commercially available fibre-strand body filler 6371 (The Martin Senour Company, Cleveland, Ohio) and left for approximately $15 \mathrm{~min}$ to set. A similar set-up has been used to test patellar tendons (Haut and Powlison, 1990; Haut and Haut, 1997) (Fig. 1). The menisci and attachments were kept moistened with saturated gauze throughout the preparation. The attachments were oriented physiologically within the fixture to replicate in situ loading conditions by aligning their collagen fibers parallel to the loading axis of the fixture.

After the filler had set, the fixture with the potted specimen inside was placed in a servo-hydraulic uniaxial materials testing machine (Model 8872, Instron Corp., Canton, Massachusetts). A custom designed "cryogrip", which supplied liquid nitrogen into the back of the clamp to freeze the portion of the meniscus within the grip, was built to grasp the meniscal tissue (Riemersa and Schamhardt, 1982; Sharkey et al., 1995; Maes and Haut Donahue, 2006). A universal joint assured that uniaxial tension was applied to the test specimens.

The cross-sectional area of the attachments was measured at the midpoint of the attachment using an area micrometer and did not appear to change along the length of the attachment (Ellis, 1969; Allard et al., 1979). The lengths of the specimens were measured using digital calipers from the insertion into the tibia to the transition between the ligamentous attachment and the meniscal tissue. The medial anterior attachment (MA), medial posterior attachment (MP), and lateral anterior attachment (LA) were tested. In contrast to the human knee where all four meniscal horn attachments insert into the tibia, the posterior attachment of the lateral meniscus in the bovine knee inserts into the femur and thus was not included in the current study.

\subsection{Mechanical testing}

Each attachment was preconditioned for 10 cycles at $10 \mathrm{~mm} / \mathrm{min}$, between $0 \%$ and $3 \%$ of the gauge length using a sine wave. Immediately following preconditioning, a pull to failure test was performed. The pull to failure tests were conducted at $2 \% / \mathrm{s}$ for all three attachments (Lam et al., 1995; Quapp and Weiss, 1998; Haut Donahue et al., 2001).

During the tests, the load, displacement, and time were recorded at $10 \mathrm{~Hz}$ using the system software (Wavemaker, INSTRON Corp., Canton, Massachusetts). As detailed below, markers were applied to the surface of the attachments and a charge-coupled video camera was used to record the pull to failure test and determine the strain distribution over the specimen surface. After preconditioning and pull to failure testing was performed on

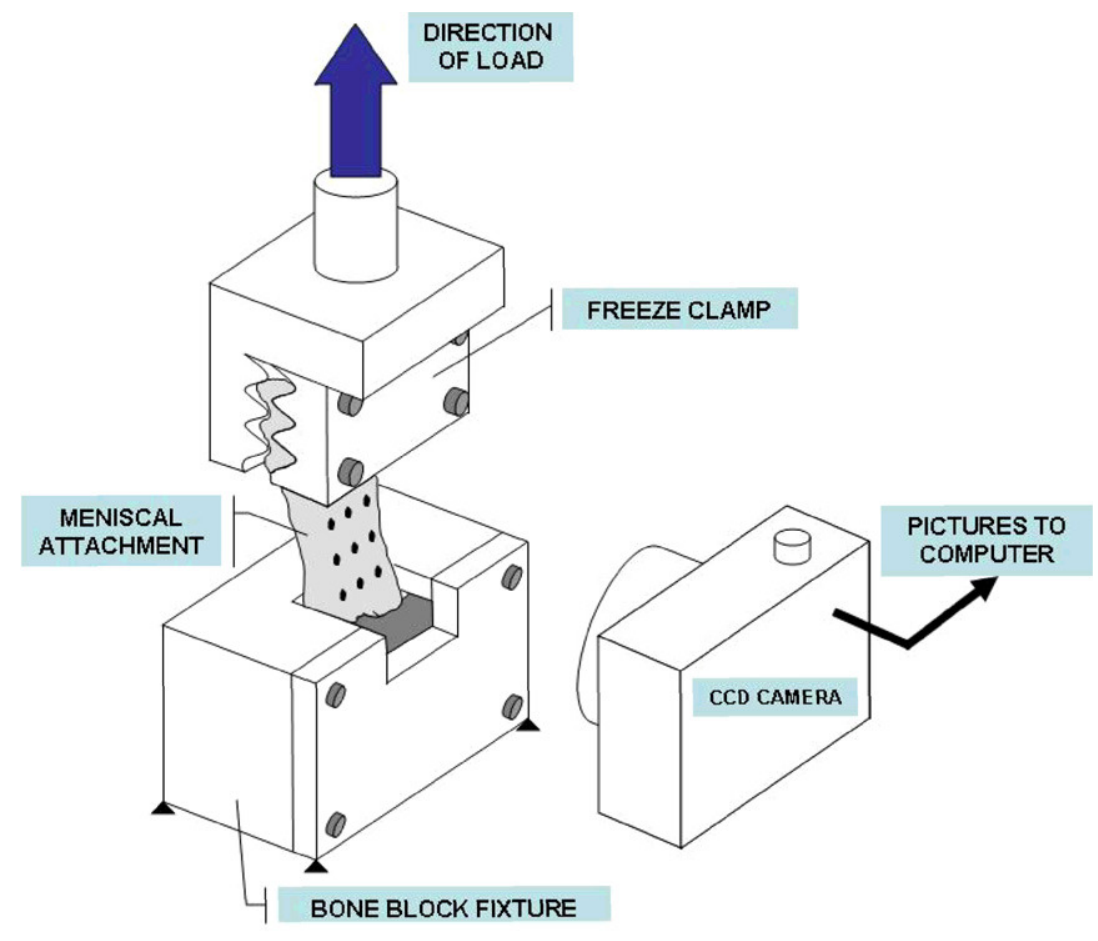

Fig. 1. Schematic of test set-up for meniscal attachments. A uniaxial tensile test was conducted at $2 \% / \mathrm{s}$ using a hydraulic testing system. 
an individual attachment, the next attachment was then placed within the fixture and tested following the same protocol. From each specimen, the three attachments were tested in random order.

To quantify the structural and material properties of the attachments, the linear stiffness $(\mathrm{N} / \mathrm{mm})$, linear modulus $(\mathrm{MPa})$, ultimate load $(\mathrm{N})$, ultimate elongation $(\mathrm{mm})$, ultimate strain $(\%)$, and ultimate stress (MPa) were determined. The stiffness and linear modulus were defined as the slope of the linear region on the load versus displacement and stress versus strain plots, respectively, and they were determined by linear regression.

\subsection{Strain distribution analysis}

A system of markers and a digital video camera were used to optically determine the strain distribution over the specimen surface. The tissue surface was wiped dry and circular markers with a diameter of approximately $500 \mu \mathrm{m}$ were applied to the surface of the tissue with waterproof Indian ink just prior to the start of the test. The markers were arranged on the surface of the attachment in a grid-like fashion with the columns and rows approximately $2 \mathrm{~mm}$ apart, thus dividing the tissue surface into four discrete regions with three longitudinal directions over which the strain could be analyzed. Region 1 was defined as the region of the attachment that inserts into the meniscal body, regions 2 and 3 were defined as the middle portion of the attachment (midsubstance), and region 4 was defined as the region of the attachment closest to the bony insertion site. The meniscal attachment surface was further sectioned into inner (i), middle (m), and outer (o) regions (Fig. 2). The inner region corresponds to the side of the attachment that corresponds to the inner radial border of the meniscal body, and the outer region corresponds to the region of the attachment that would adjoin the outer radial periphery of the meniscal main body. The meniscal attachments were divided along the length into three regions to be consistent with previous studies of ligamentous attachments in the knee. The attachments were further divided into inner and outer segments based on studies which suggest that the mechanical environment within the meniscus (Spilker et al., 1992; LeRoux et al., 2001) varies from the inner region to the outer region. To aid in marker placement, a template was used to insure consistency across all samples tested. A charge-couple video camera (model MicroPix M1024, 0.786 Mega pixels, CCD-Direct, Ann Arbor, Michigan) recorded, in real time, the motion of the markers by taking still pictures at $10 \mathrm{~Hz}$. Pictures captured from the digital video camera were analyzed using a custom-made processing program (Matlab, version 7.1.0.246 (R14)). The program changed the contrast of the picture in order to emphasize the markers on the surface. The centroid of each marker on every picture was

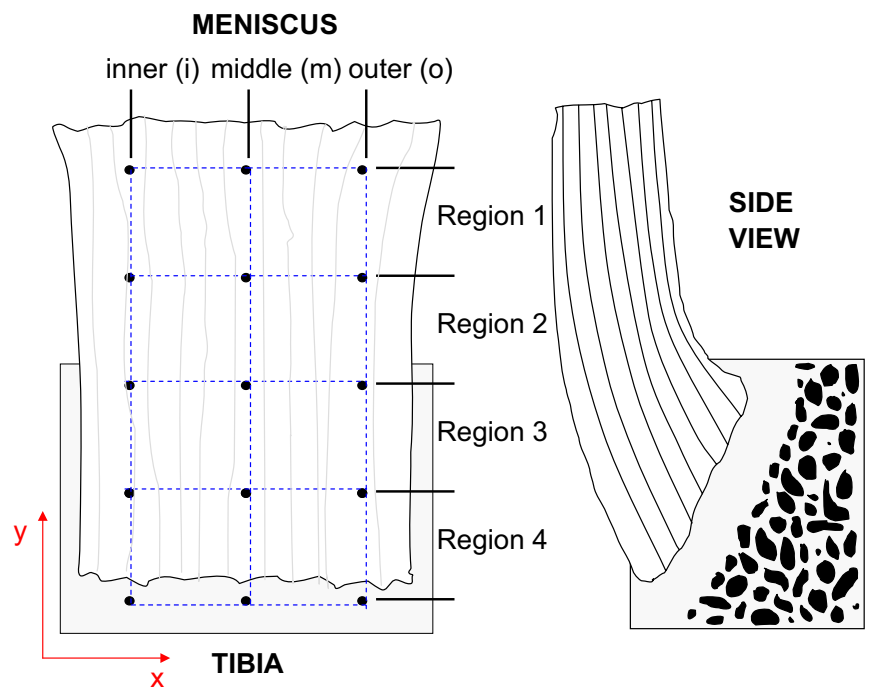

Fig. 2. Picture of the marker dot grid placed on the tissue surface and the distinct regions and longitudinal directions created. found using the program. The distance in pixels between centroids was measured and stored to calculate the strain in each region. Strains were calculated using Green's strain because strains were on the order of $30 \%$ (Crisfield, 1991). The mode of failure was classified as failure at the gripping interface, mid-substance failure, or bone avulsion.

\subsection{Statistical analyses}

Chauvenet's criterion was used to reject extraneous data. Means and standard deviations were computed for the geometric properties (crosssectional area, length), structural properties (ultimate load, ultimate displacement, linear stiffness), and the ultimate stress for each of the three attachments. One-way analysis of variance (ANOVA) was performed using Statview (SAS Institute Inc., Cary, North Carolina) to make comparisons of the geometric and structural properties and the ultimate stress between the three attachments. When significant results were identified by one-way ANOVA, post hoc comparisons were made using Bonferroni-Dunn's method $(p<0.05)$. A three factor (attachment, region, longitudinal section) ANOVA was performed to compare the linear moduli and ultimate strains between the attachments and between the four regions and three longitudinal sections. Post hoc comparisons were made using Bonferroni-Dunn's method when significant results were identified. A significance level of 0.05 was used for all statistical analyses. A post doc power analysis was completed for data showing no significance. Based on a previous study of the sensitivity of tibio-femoral contact mechanics to the properties of meniscal horn attachments, $\Delta$, which is the magnitude of the minimum range which is important to detect for a given parameter, was selected to be $200 \mathrm{~N} / \mathrm{mm}$ for the stiffness of the attachment. For stiffness, this created a $\Delta / \sigma$ of 2.00 . Since data were not available for the other parameters of interest, we consistently used $\Delta / \sigma$ of 2.00 to compute the power for all analysis.

\section{Results}

The LA attachment was significantly longer than the MA attachment $(p=0.0004)$ and the MP attachment $(p<0.0001$, Table 1$)$. The MA attachment was also significantly longer than the MP attachment $(p<0.0001$, Table 1). There were no statistically significant differences in cross-sectional area between the attachments tested $(p>0.05$, power $=90 \%$, Table 1$)$.

The linear stiffness had correlation coefficients of 0.93 or greater for all three attachments. The ultimate elongation showed less than a $6 \%$ variation between all three attachments (Fig. 3, Table 1). There were no significant differences between the attachments for the ultimate load, ultimate elongation, linear stiffness, or ultimate stress $(p>0.05$, power $>90 \%)$. Ultimate stress in the LA, MA, and MP attachments were $66.2 \pm 23.8,62.7 \pm 15.6$, and $76.2 \pm 28.2 \mathrm{MPa}$, respectively.

Both local ultimate strains and local linear moduli were calculated for each region, across all attachments (Figs. 4-6). No significant differences were found between regions (1-4) or longitudinal sections (i, $\mathrm{m}, \mathrm{o}$ ) for either the ultimate strain or linear modulus $(p>0.05$, power $=80 \%$ for both linear modulus and ultimate strain). However, the three factor ANOVA showed significant differences between the attachments. The linear modulus of the MA $(154 \pm 134 \mathrm{MPa})$ was significantly less than both the MP $(248 \pm 179 \mathrm{MPa}, p=0.0111)$ and the LA $(281 \pm 214 \mathrm{MPa}$, $p=0.0007)$. Likewise, the ultimate strain for the MA 
Table 1

Structural properties for the lateral anterior (LA), medial anterior (MA), and medial posterior (MP) meniscal horn attachments

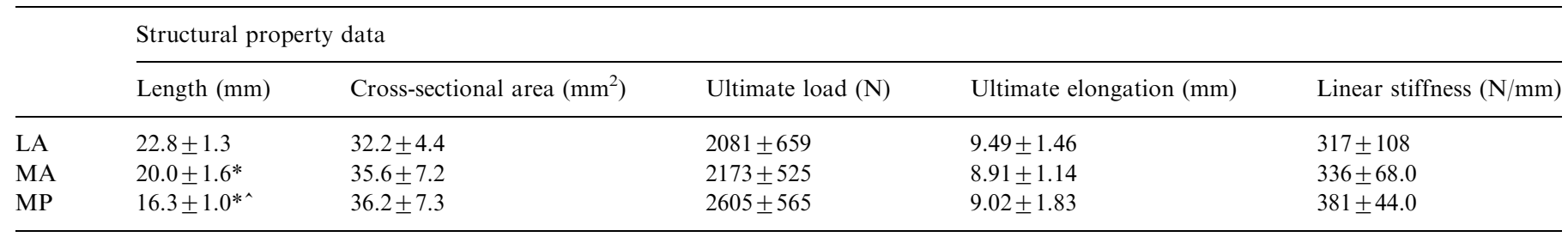

Properties measured across all regions of the attachments.

* Significantly different than LA $(p<0.05)$.

S Significantly different than MA $(p<0.05)$.

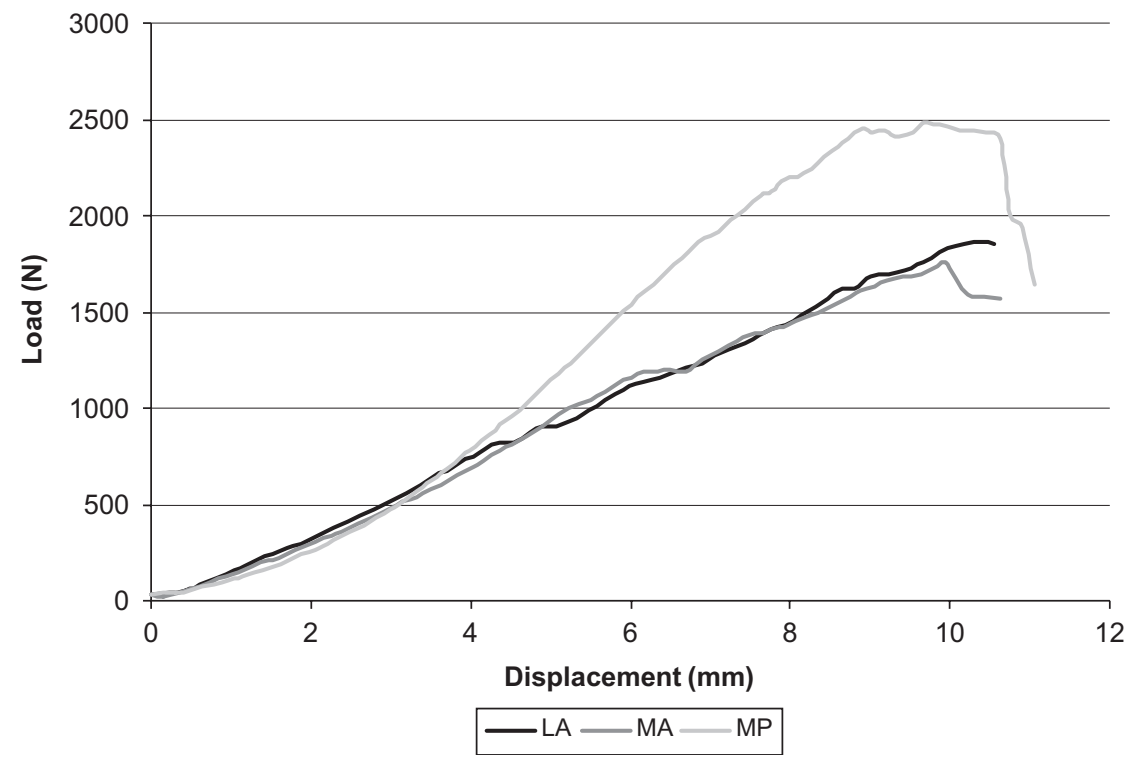

Fig. 3. Sample load-displacement plot illustrating the similarities of the ultimate loads and elongations. The ultimate loads and elongations of all three attachments showed no significant differences.

$(13.5 \pm 8.8 \%)$ was significantly less than the MP $(23 \pm 13 \%$, $p<0.0001)$ and the LA $(20.3 \pm 11.1 \%, p=0.0033)$.

While no significant differences were noted across the surface of the attachments, some general trends were noted. Region 4 (insertion to bone) showed the largest ultimate strains across the regions for the LA and MA attachments (Figs. 4 and 5). The opposite trend was seen for the MP attachment which had lower strains in region 4, with strains increasing up to region 1 (Fig. 6). The linear modulus in the LA $(84.2 \pm 29.1 \mathrm{MPa})$ and MP $(128 \pm 87 \mathrm{MPa})$ attachments were lowest in region 4, middle zone. The greatest linear modulus $(559.4 \pm 441.7 \mathrm{MPa})$ was in the midsubstance (region 3) for the LA attachment, specifically in the inner section, while conversely, the modulus of the MA attachment was lowest in region 3 for all sections.

For all attachments and regions, the largest strains were always on either the inner or outer section, never in the middle section. Mid-substance failure occurred in 22 of the 24 attachments tested while the remaining two failed by avulsion. The mid-substance failures were always in region 3 or 4 .

\section{Discussion}

The research presented in this manuscript is timely for many reasons. In the design of meniscal replacements, including tissue engineered menisci, anchoring of the replacement is necessary (Setton et al., 1999; Haut Donahue and Maes, 2006). The data presented documents the material properties of individual meniscal attachments and makes comparisons between the attachments, noting statistical differences in modulus and ultimate strain. To date, only one set of experimental data is available documenting any mechanical behavior of the meniscal attachments (Goertzen et al., 1996) and these data only include failure loads. Not only is this data important for the design of meniscal replacements, but previous computational models have documented the sensitivity of knee joint contact behavior and kinematics on the material properties of the meniscal attachments (Haut Donahue et al., 2003; Yao et al., 2006). For total knee joint finite element models to accurately represent the behavior of the knee, including true motion of the menisci, material property data for meniscal attachments is necessary 


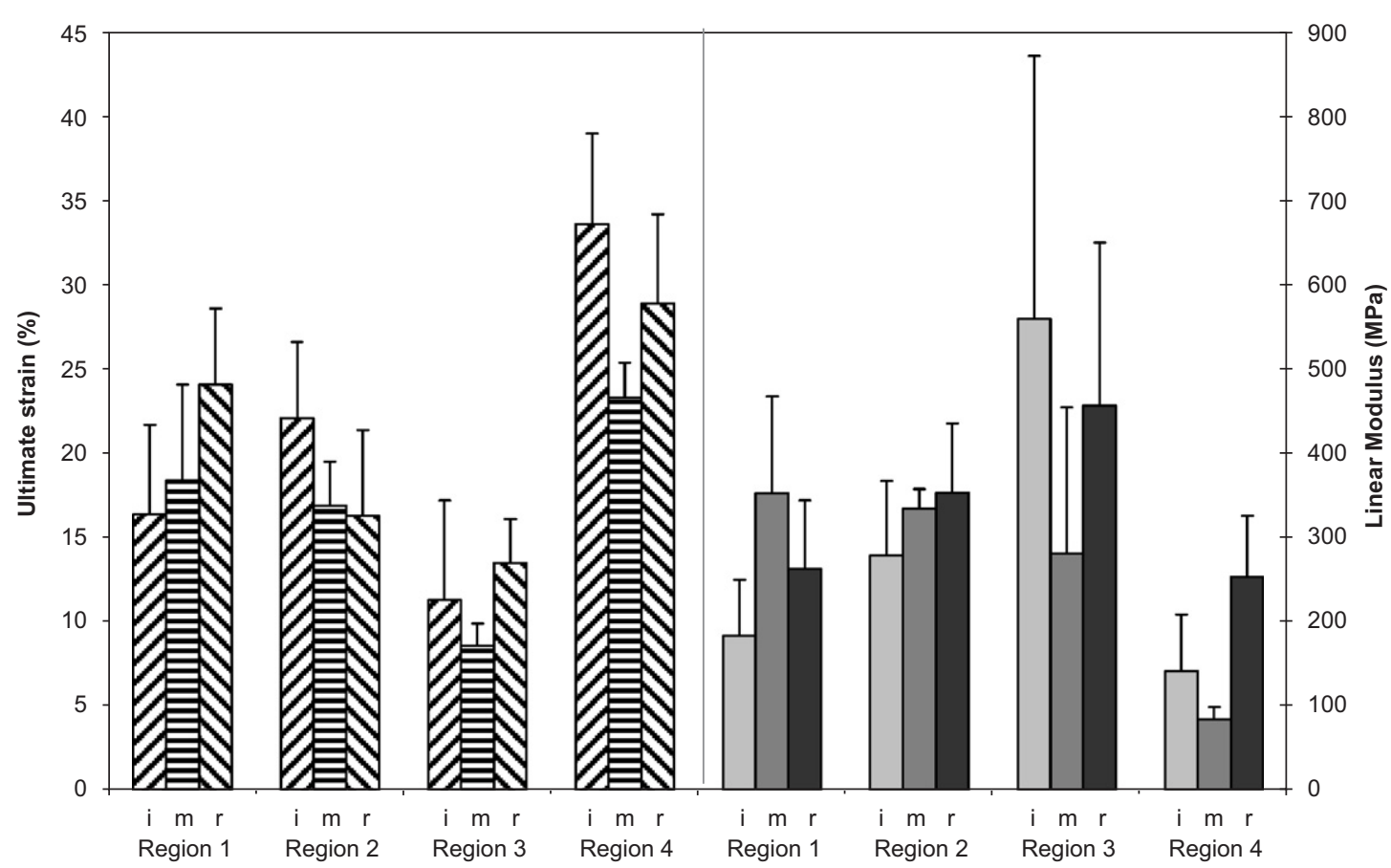

Fig. 4. Ultimate strain and linear modulus in the LA attachment. Region 1 is closest to the grip interface, regions 2 and 3 are mid-substance, and region 4 is near the bone insertion site. Longitudinal sections were inner (i), middle (m), and outer (o). Data are presented as mean \pm standard deviation.

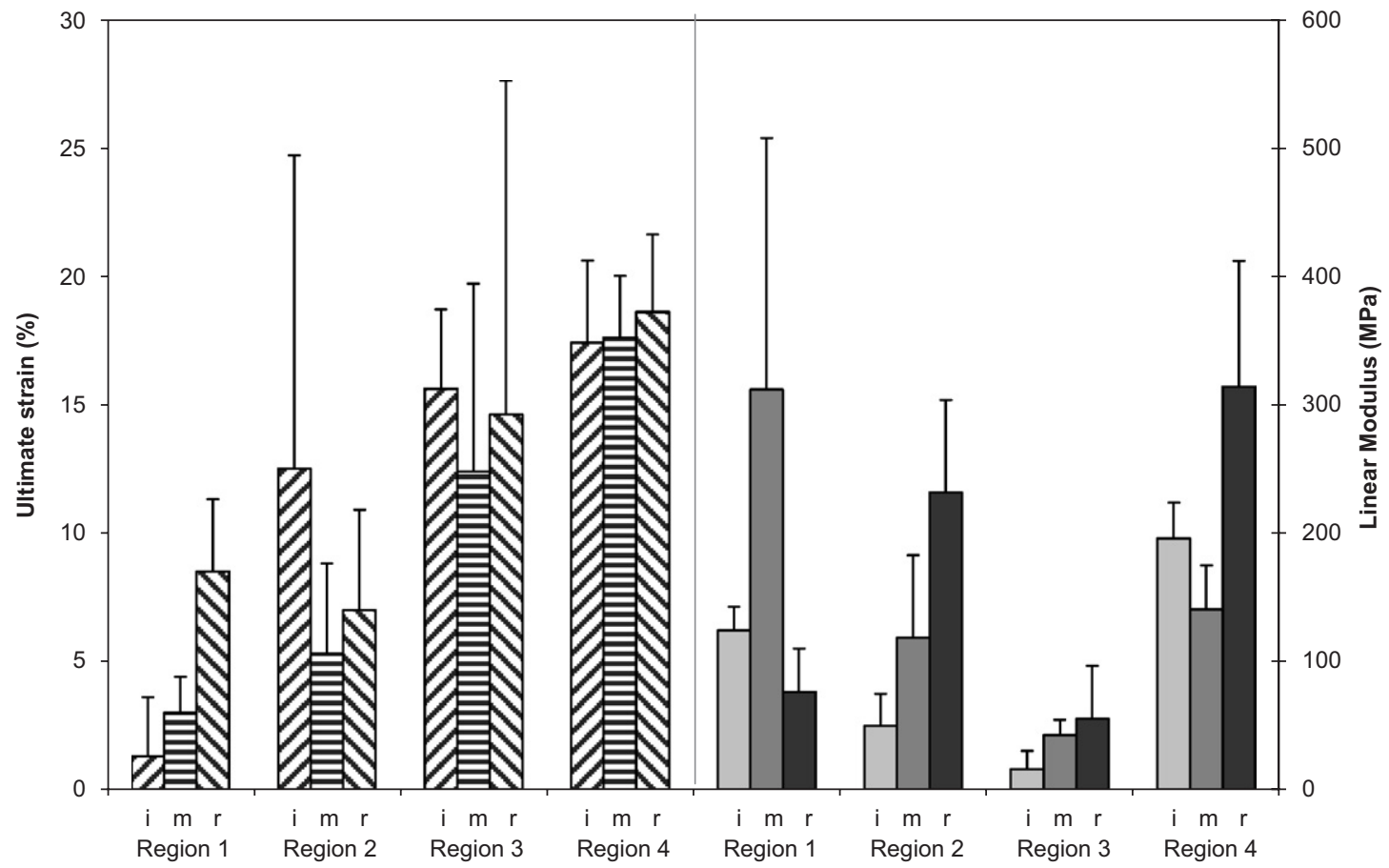

Fig. 5. Ultimate strain and linear modulus of the MA attachment. Region 1 is closest to the grip interface, regions 2 and 3 are mid-substance, and region 4 is near the bone insertion site. Longitudinal sections were inner (i), middle (m), and outer (o). Data are presented as mean \pm standard deviation.

(Yao et al., 2006). Furthermore, in a review article on tissue engineered meniscal repair, Setton et al. (1999) calls for the properties of the meniscal attachments.

While not significant, the MP attachment exhibited a larger stiffness and ultimate load than the MA and LA attachments. This correlates to the posterior attachments resistance to movement in vivo and the associated increase in contact stresses on the posterior region of the meniscus. The LA and MP attachments have a higher modulus and ultimate strain compared to the MA attachment, suggesting that the LA and MP attachment exhibited higher strain energy. It is possible that these attachments with higher 


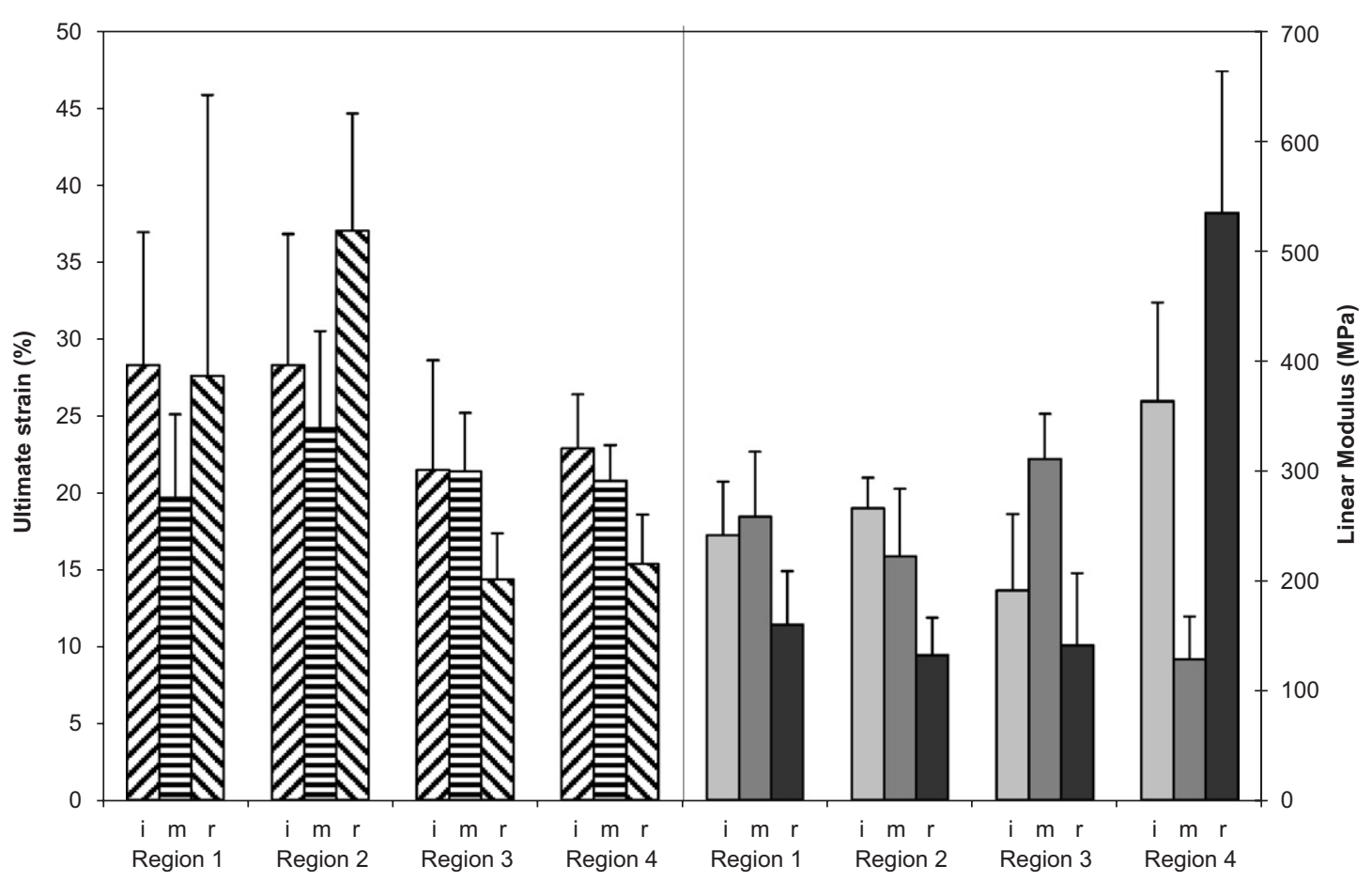

Fig. 6. Ultimate strain and linear modulus in the MP attachment. Region 1 is closest to the grip interface, regions 2 and 3 are mid-substance, and region 4 is near the bone insertion site. Longitudinal sections were inner (i), middle (m), and outer (o). Data are presented as mean \pm standard deviation.

strain energy are able to support higher loads compared to the MA attachment. When designing a meniscal replacement, attention needs to be paid to the varying properties of the various attachments.

\subsection{Limitations of study}

A previous study conducted to measure the material properties of the meniscal attachments using a rabbit model documented the peak failure load with no documentation of cross-sectional areas or failure mode (Goertzen et al., 1996). The bovine knee model is closer in size to a human knee than a rabbit knee, and bovine tendon properties have been shown to correlate well to human tendon properties (Haut Donahue et al., 2001). Sweigart et al. (2004) shows fairly good agreement between bovine meniscal tissue (0.11-0.21 MPa) and human meniscal tissue $(0.09-0.16 \mathrm{MPa})$ for the aggregate modulus (Sweigart et al., 2004). Additionally, Joshi et al. (1995) in a study of five animal models compared to human meniscal tissue showed that the permeability of the bovine meniscus was not statistically different than the human meniscus. However, a limitation of the bovine model, as well as the rabbit model, is that the posterior attachment of the lateral meniscus inserts into the femur, while in the human knee joint, this attachment inserts into the tibia.

\subsection{Significance of results}

The results of this study indicated that the medial anterior meniscal attachment had significantly smaller ultimate strains and moduli compared to the remaining two attachments. In the human knee the anterior horns have been shown to translate more than the posterior horns during knee motion (Thompson et al., 1991; Vedi et al., 1999). While it remains unknown if bovine and human menisci have the same pattern of motion, the increased stiffness and modulus found in this current study of the bovine posterior attachment may help explain the reduced displacements of human menisci during knee motion. The posterior attachment's resistance to movement may relate to clinical observations of greater damage occurring in the posterior region of the meniscus because the reduced ability for translation leads to larger contact stresses in this region (Walker and Erkman, 1975; Wyland et al., 2002). In addition, during knee flexion the load of the posterior aspect of the femoral-tibial area is increased, likely translating to increased loads on the posterior meniscal attachment. Therefore, it is likely that the loading environment of the meniscal attachments influences their structural and material properties.

Using a video dimension analysis system, higher strains have been measured at the bony insertion than in the midsubstance for many knee ligaments (Woo et al., 1983; Butler et al., 1984; Lam et al., 1995; Gardiner et al., 2001; Hirokawa et al., 1998). Gao et al. (1996) found that avulsion or avulsion fracture was a common mode of failure for various soft connective tissues, which correlates to the findings of higher strains at the bony insertion sites. The current study showed that while not significant, both anterior attachments (lateral and medial) had larger local strains near the bony insertion for a strain rate of $2 \% / \mathrm{s}$. In 
contrast, the medial posterior attachment tended to have larger strains near the meniscal body versus the bony insertion at a strain rate of $2 \% / \mathrm{s}$. Perhaps these differences are related to the different in vivo loading environments.

The clinical implications of the results indicate that a meniscal replacement would require the horn attachments to possess varying material properties. It is possible that if constant material properties were used for all three attachments, joint laxity may be altered thereby decreasing the ability of the meniscus to provide a transition from the round femoral condyles to the flat tibial plateau.

The findings of this study document the failure properties of the meniscal horn attachments, as well as the surface strain distribution. The failure properties obtained, along with the time dependent properties documented previously by the current authors, provide additional insight into designing and implementing a successful meniscal replacement (Maes and Haut Donahue, 2006).

\section{Acknowledgment}

This work was funded by the National Institutes of Health (AR051906-01).

\section{References}

Ahmed, A.M., 1992. The load bearing role of the knee menisci. In: Mow, V.C., Arnoczky, S., Jackson, D. (Eds.), Knee Meniscus-Basic and Clinical Foundations. New York, Raven Press, pp. 59-73.

Alhalki, M.M., Howell, S.M., et al., 1999. How three methods for fixing a medial meniscal autograft affect tibial contact mechanics. American Journal of Sports Medicine 27 (3), 320-328.

Allard, P., Thiry, P.S., et al., 1979. Pressure dependence of "the area micrometer" method in evaluation of cruciate ligament cross-section. Journal of Biomedical Engineering 1 (4), 265-267.

Allen, P.R., Denham, R.A., et al., 1984. Late degenerative changes after meniscectomy - factors effecting the knee after operation. British Journal of Bone and Joint Surgery 66, 666-671.

Asik, M., Sener, N., 2002. Failure strength of repair devices versus meniscus suturing techniques. Knee Surgery Sports Traumatology Arthroscopy 10 (1), 25-29.

Butler, D.L., Grood, E.S., et al., 1984. Effects of structure and strain measurement technique on the material properties of young human tendons and fascia. Journal of Biomechanics 17 (8), 579-596.

Chen, M.I., Branch, T.P., et al., 1996. Is it important to secure the horns during lateral meniscal transplantation? A cadaveric study. Arthroscopy 12 (2), 174-181.

Crisfield, M.A., 1991. Non-Linear Finite Element Analysis of Solids and Structures. Wiley, NewYork.

DeHaven, K.E., 1992. Meniscectomy versus repair: clinical experience. In: Mow, V.C., Arnoczky, S.P., Jackson, D.W. (Eds.), Knee Meniscus: Basic and Clinical Foundations. Raven Press Ltd., New York, NY, pp. 131-141.

Ellis, D.G., 1969. Cross-sectional area measurments for tendon specimens: a comparision of several methods. Journal of Biomechanics 2, 175-186.

Fithian, D.C., Kelly, M.A., et al., 1990. Material properties and structurefunction relationships in the menisci. Clinical Orthopedics and Related Research 252, 19-31.

Gao, J., Rasanen, T., et al., 1996. The morphology of ligament insertions after failure at low strain velocity: an evaluation of ligament entheses in the rabbit knee. Journal of Anatomy 189 (part 1), 127-133.
Gao, J., Wei, X., et al., 1998. Healing of the anterior attachment of the rabbit meniscus to bone. Clinical Orthopedics 348, 246-258.

Gardiner, J.C., Weiss, J.A., et al., 2001. Strain in the human medial collateral ligament during valgus loading of the knee. Clinical Orthopedics and Related Research 391, 266-274.

Ghadially, F.N., Wedge, J.H., et al., 1986. Experimental methods of repairing injured menisci. Journal of Bone and Joint Surgery. British Volume 68 (1), 106-110.

Ghosh, P., Taylor, T.K., 1987. The knee joint meniscus. A fibrocartilage of some distinction. Clinical Orthopedics 224, 52-63.

Goertzen, D., Gillquist, J., et al., 1996. Tensile strength of the tibial meniscal attachments in the rabbit. Journal of Biomedical Materials Research 30 (1), 125-128.

Haut Donahue, T.L., Maes, J.A., 2006. Meniscal Replacements. Wiley Encyclopedia of Biomedical Engineering. In: Akay, M. (Ed.). Wiley, Hoboken.

Haut Donahue, T.L., Gregersen, C., et al., 2001. Comparison of viscoelastic, structural and material properties of the double-looped anterior cruciate ligament grafts made from bovine digital extensor and human hamstring tendons. Journal of Biomechanical Engineering 123 (2), 162-169.

Haut Donahue, T.L., Hull, M.L., et al., 2003. How the stiffness of meniscal attachments and meniscal material properties affect tibiofemoral contact pressure computed using a validated finite element model of the human knee joint. Journal of Biomechanics 36 (1), 19-34.

Haut, R.C., Powlison, .C., 1990. The effects of test environment and cyclic stretching on the failure properties of human patellar tendons. Journal of Orthopedic Research 8 (4), 532-540.

Haut, T.L., Haut, R.C., 1997. The state of tissue hydration determines the strain-rate-sensitive stiffness of human patellar tendon. Journal of Biomechanics 30 (1), 79-81.

Hirokawa, S., Yamamoto, K., et al., 1998. A photoelastic study of ligament strain. IEEE Transactions Rehabilitation Engineering 6 (3), 300-308.

Joshi, M.D., Suh, J.K., et al., 1995. Interspecies variation of compressive biomechanical properties of the meniscus. Journal of Biomedical Matererials Research 29 (7), 823-828.

Lam, T.C., Shrive, N.G., et al., 1995. Variations in rupture site and surface strains at failure in the maturing rabbit medial collateral ligament. Journal of Biomechanical Engineering 117 (4), 455-461.

LeRoux, M. A., Upton, M. L., et al., 2001. Biphasic finite element modeling of tear effects on the mechanics of the meniscus. ASMEBED 50.

Maes, J.A., Haut Donahue, T.L., 2006. Time dependent properties of bovine meniscal attachments: stress relaxation and creep. Journal of Biomechanics 39 (16), 3055-3061.

McBride, I.D., Reid, J.G., 1988. Biomechanical considerations of the menisci of the knee. Canadian Journal of Sports Science 13 (4), 175-187.

Messner, K., 1999. Meniscal regeneration or meniscal transplantation. Scandinavian Journal of Medicine Science and Sports 9 (3), 162-167.

Messner, K., Gao, J., 1998. The menisci of the knee joint. Anatomical and functional characteristics, and a rationale for clinical treatment. Journal of Anatomy 193 (Pt 2), 161-178.

Moon, M.S., Woo, Y.K., et al., 1988. Meniscal regeneration and its effects on articular cartilage in rabbit knees. Clinical Orthopedics and Related Research 227, 298-304.

Morrison, J.B., 1970. The mechanics of the knee joint in relation to normal walking. Journal of Biomechanics 3 (1), 51-61.

Newman, A.P., Daniels, A.U., et al., 1993. Principles and decision making in meniscal surgery. Arthroscopy 9 (1), 33-51.

Paletta, G.A., Manning, T., et al., 1997. The effect of allograft meniscal replacement on intraarticular contact area and pressures in the human knee. American Journal of Sports Medicine 25 (5), 692-698.

Quapp, K.M., Weiss, J.A., 1998. Material characterization of human medial collateral ligament. Journal of Biomechanical Engineering 120 (6), 757-763. 
Renstrom, P., Johnson, R.J., 1990. Anatomy and biomechanics of the menisci. Clinincs in Sports Medicine 9 (3), 523-538.

Riemersa, D.J., Schamhardt, H.C., 1982. The cryo-jaw, a clamp designed for in vitro rheology studies of horse digital flexor tendons. Journal of Biomechanics 15 (8), 619-620.

Rodeo, S.A., 2001. Meniscal allografts - where do we stand? American Journal of Sports Medicine 29 (2), 246-261.

Sekaran, S.V., Hull, M.L., et al., 2002. Nonanatomic location of the posterior horn of a medial meniscal autograft implanted in a cadaveric knee adversely affects the pressure distribution on the tibial plateau. American Journal of Sports Medicine 30 (1), 74-82.

Setton, L.A., Guilak, F., et al., 1999. Biomechanical factors in tissue engineered meniscal repair. Clinical Orthopedics 367 (Suppl), S254-S272.

Sharkey, N.A., Smith, T.S., et al., 1995. Freeze clamping musculotendinous junctions for in vitro simulation of joint mechanics. Journal of Biomechanics 28 (5), 631-635.

Shrive, N.G., JJ, O.C., et al., 1978. Load-bearing in the knee joint. Clinical Orthopedics 131, 279-287.

Spilker, R.L., Donzelli, P.S., et al., 1992. A transversely isotropic biphasic finite element model of the meniscus. Journal of Biomechanics 25 (9), $1027-1045$.
Sweigart, M.A., Zhu, C.F., et al., 2004. Intraspecies and interspecies comparison of the compressive properties of the medial meniscus. Annals of Biomedical Engineering 32 (11), 1569-1579.

Thompson, W.O., Thaete, F.L., et al., 1991. Tibial meniscal dynamics using three-dimensional reconstruction of magnetic resonance images. American Journal of Sports Medicine 19 (3), 210-215.

Vedi, V., Williams, A., et al., 1999. Meniscal movement. An in-vivo study using dynamic MRI. Journal of Bone and Joint Surgery 81 (1), 37-41.

Walker, P.S., Erkman, M.J., 1975. The role of the menisci in force transmission across the knee. Clinical Orthopedics 109, 184-192.

Woo, S.L., Gomez, M.A., et al., 1983. Measurement of mechanical properties of ligament substance from a bone-ligament-bone preparation. Journal of Orthopedic Research 1 (1), 22-29.

Wyland, D.J., Guilak, F., et al., 2002. Chondropathy after meniscal tear or partial meniscectomy in a canine model. Journal of Orthopedic Research 20 (5), 996-1002.

Yao, J., Funkenbusch, P.S., et al., 2006. Sensitivities of medial meniscal motion and deformation to material properties of articular cartilage, meniscus and meniscal attachments using design of experiments methods. Journal of Biomechanical Engineering 128 (3), 399-408. 